\title{
THE PROBABILITY OF CONNECTEDNESS OF AN UNLABELLED GRAPH CAN BE LESS FOR MORE EDGES
}

\author{
E. M. WRIGHT ${ }^{1}$
}

\begin{abstract}
We write $\beta=\beta(n, q)$ for the probability that a graph on $n$ unlabelled nodes with $q$ edges is connected; that is $\beta$ is the ratio of the number of connected graphs to the total number of graphs. We write $N=n(n-1) / 2$. For fixed $n$ we might expect that $\beta$ would increase with $q$, at least nonstrictly. On the contrary, we show that, for any given integer $s$, we have $\beta(n, q+1)<\beta(n, q)$ for $N-n-s \leqq q \leqq N-n$ and $n>n_{0}(s)$. We can show that $\beta(n, q+1)<\beta(n, q)$ for a much longer range, but this requires much more elaborate arguments.
\end{abstract}

An $(n, q)$ graph has $n$ nodes and $q$ edges, where each edge is a different unordered pair of nodes. We write $N=n(n-1) / 2$, so that $0 \leqq q \leqq N$. We write $F_{n q}$ (resp. $\left.T_{n q}\right)$ for the number of $(n, q)$ graphs in which the nodes are labelled (resp. unlabelled) and $f_{n q}$ (resp. $t_{n q}$ ) for the number of these graphs which are connected. Then $\alpha_{n q}=f_{n q} / F_{n q}$ (resp. $\beta_{n q}=t_{n q} / T_{n q}$ ) is the probability that a labelled (resp. unlabelled) $(n, q)$ graph is connected. It is natural to expect that, for fixed $n$, these probabilities will increase (in the nonstrict sense) as $q$ increases. For $\alpha_{n q}$, this is true and can be proved trivially. For $\beta_{n q}$ it is false; the simplest counterexample is that $\beta_{69}=$ $20 / 21$ and $\beta_{6,10}=14 / 15$ (see, for example, the diagrams of all $(6,9)$ and $(6,10)$ graphs in $[6])$.

The slightly surprising phenomenon that $\beta_{n q}$ can decrease as $q$ increases seems worth further study. Here we prove the following theorem.

THEOREM. For any positive integer $v$, there is an $n_{0}=n_{0}(v)$, such that

$$
\beta_{n, \nu-n-s}>\beta_{n, N-n-s+1} \quad(0 \leqq s \leqq v)
$$

when $n>n_{0}$ and, indeed, such that

$$
1-\beta_{n, N-n-s+1}>C n^{1 / 2}\left(1-\beta_{n, N-n-s}\right) .
$$

Presented to the Society, September 15, 1971 under the title Decreasing probability of connectedness of a graph as number of edges increases; received by the editors October 9 , 1971.

AMS 1969 subject classifications. Primary 0565.

Key words and phrases. Unlabelled graphs, probability of connectedness.

1 The research reported herein has been spunsored by the United States Government. 
Thus, for these values of $q, \beta_{n q}$ retreats rapidly from 1 as $q$ increases. Every statement in what follows is subject to the condition "for large enough $n$."' The positive number $C$, not always the same at each occurrence, is independent of $n$ and $q$.

For the moment we consider only unlabelled graphs. Any disconnected $(n, q)$ graph consists of a $\left(k, q_{1}\right)$ graph together with a $\left(n-k, q-q_{1}\right)$ graph for some $q_{1}$ and for some $k$ (not necessarily unique) such that $1 \leqq k \leqq n / 2$. Compared with the complete $(n, N)$ graph, the disconnected $(n, q)$ graph lacks at least $k(n-k)$ edges, so that $q \leqq N-k(n-k)$. If $q=N-n-s$, where $-1 \leqq s \leqq n-5$, we have $k=1$. Hence a disconnected $(n, N-n-s)$ graph has just 2 components, one an isolated node and the other an $(n-1, N-n-s)$ graph. The latter graph, compared with the complete $(n-1,(n-1)(n-2) / 2)$ graph, lacks $\{(n-1)(n-2) / 2\}-(N-n-s)=s+1$ edges. Hence it is the complement of an $(n-1, s+1)$ graph. The relation is $(1,1)$ and so

$$
T_{n, N-n-s}-t_{n, V-n-s}=T_{n-1, s+1} \quad(-1 \leqq s \leqq n-5) .
$$

Clearly $T_{n-1,0}=T_{n-1,1}=1$ and so $\beta_{n, N-n+1}<\beta_{n, N-n}$ provided $T_{n, N-n+1}<$ $T_{n, \mathrm{~V}-n}$, i.e. provided $T_{n, n-1}<T_{n, n}$. The smallest $n$ for which this is true is 6 , where $T_{65}=15, T_{66}=21$ (see [6]). Hence $\beta_{6,10}<\beta_{69}$, the counterexample given above.

If $n \geqq 2 q$, the $(n, q)$ graphs can be put into $(1,1)$ correspondence with the $(2 q, q)$ graphs by removing $n-2 q$ of the isolated nodes from each of the former and conversely. Hence $T_{n q}=T_{2 q, q}$ and, from (3), we have

$$
T_{n, N-n-s}-t_{n, N-n-s}=T_{2(s+1), s+1} \quad(-1 \leqq s \leqq v) .
$$

To prove (2), we have then to show that

$$
T_{2 s, s} / T_{n, N-n-s+1}>C n^{1 / 2} T_{2 s+2, s+1} / T_{n, N-n-s} .
$$

Since $T_{2 s+2, s+1} / T_{2 s, s}=C$, it is enough to show that $T_{n, N-n-s}>$ $C n^{1 / 2} T_{n, N-n-s+1}$, that is $T_{n, n+s}>C n^{1 / 2} T_{n, n+s-1}$.

We shall prove a little more, viz. the following lemma.

Lemma. If $C n<q<C n$, then $T_{n, q+1}>C n^{1 / 2} T_{n q}$.

Henceforth we suppose the hypothesis of the lemma to be satisfied. We now use Polya's famous counting theorem [1], [5], [8]. $S_{n}$ is the symmetric group (of degree $n$ and order $n$ !) of all the permutations $\pi$ of the $n$ labelled nodes in a labelled $(n, q)$ graph, and $F_{\pi}=F_{n}(n, q)$ is the number of labelled $(n, q)$ graphs invariant under $\pi$. Then Polya's theorem tells us that

$$
n ! T_{n q}=\sum_{\pi \in S_{n}} F_{\pi}
$$


We are henceforth concerned with the enumeration of labelled graphs. The permutation $\pi$ has a unique expression as a product of disjoint cycles, which contains $p_{j}$ node-cycles of length $j$, where $1 \leqq j \leqq n$. The node permutation $\pi$ induces a permutation on the $N$ possible edges in the graph which contains $P_{j}$ edge-cycles of length $j$. We have

$$
\sum_{j=1}^{n} j p_{j}=n, \quad \sum_{j=1}^{n} j P_{j}=N .
$$

Oberschelp [7] gives formulae for $P_{j}$ in terms of the $p_{j}$. All we require here is to note that

$$
P_{1}=\frac{1}{2} p_{1}\left(p_{1}-1\right)+p_{2} .
$$

Any graph invariant under $\pi$ must contain all the edges of a particular edge-cycle or none. Hence

$$
F_{\pi}(n, q)=\sum^{\prime} \prod_{j=1}^{n} B\left(P_{j}, s_{j}\right),
$$

where $B(h, k)=h ! /\{k !(h-k) !\}, B(h, 0)=1$ and $\Sigma^{\prime}$ denotes summation over all $s_{1}, \cdots, s_{n}$ such that $\sum j s_{j}=q$. We write $M=n^{3 / 4}$ and separate the sum on the right-hand side of (4) into two parts, so that

$$
n ! T_{n q}=T_{1}(q)+T_{2}(q)
$$

where $T_{1}(q)$ contains all the $F_{\pi}$ for which $p_{1}>M$ and $T_{2}(q)$ those for which $p_{1} \leqq M$.

First we take $p_{1}>M$, so that, $P_{1}>C n^{3 / 2}$. In (7), we replace $q$ by $q+1$, discard those terms on the right in which $s_{1}=0$ and replace $s_{1}$ by $s_{1}+1$ in the others. We have then

where

$$
F_{n}(n, q+1) \geqq \sum^{\prime} \lambda\left(P_{1}, s_{1}\right) \prod B\left(P_{j}, s_{j}\right) \geqq C n^{1 / 2} F_{\pi}(n, q),
$$

$$
\lambda\left(P_{1}, s_{1}\right)=\left(P_{1}-s_{1}\right) /\left(s_{1}+1\right) \geqq\left(C n^{3 / 2}-q\right) /(q+1) \geqq C n^{1 / 2}
$$

under the hypothesis of the lemma. Hence

$$
T_{1}(q+1)>C n^{1 / 2} T_{1}(q) .
$$

We write $[R(X)]_{q}$ for the coefficient of $X^{q}$ in the polynomial $R(X)$. From (7),

$$
F_{\pi}(n, q)=\left[\prod\left(1+X^{j}\right)^{P^{\prime}}\right]_{l} \leqq Y^{-q} \prod\left(1+Y^{j}\right)^{P_{j}},
$$

where $Y$ is any positive number. Again, if $j>2$, we have $\left(1+Y^{j}\right)^{2} \leqq$ $\left(1+Y^{2}\right)^{j}$. Hence

$$
\prod\left(1+Y^{j}\right)^{P_{j}} \leqq(1+Y)^{I_{1}}\left(1+Y^{2}\right)^{\left(N-P_{1}\right) / 2}
$$


by (5). We now put $Y=(\{q /(N-q)\})^{1 / 2}$ and have $F_{\pi}(n, q) \leqq V^{P^{3}} W$, where

and

$$
V^{2}=(1+Y)^{2} /\left(1+Y^{2}\right) \leqq 1+C n^{-1 / 2}
$$

$$
W=Y^{-q}\left(1+Y^{2}\right)^{N / 2}=(N / q)^{q / 2}\{N /(N-q)\}^{(N-q) / 2}
$$

If $p_{1} \leqq M$ we have $P_{1} \leqq C n^{3 / 2}$ and $P_{1} \log V<C n$. Again

$$
\begin{aligned}
\log W & \leqq(q / 2) \log (N / q)+\{(N-q) / 2\} \log (1+\{q /(N-q)\}) \\
& \leqq(q / 2) \log q+C n
\end{aligned}
$$

Hence $\log F_{\pi}(n, q) \leqq(q / 2) \log q+C n$. There are less than $n !$ terms in $T_{2}(q)$ and so

$$
T_{2}(q) \leqq n ! q^{q / 2} e^{C n}
$$

We now write $p=[(2 q) / \log (2 q)]$ so that $M<p \leqq n$. The number of $\pi$ for which $p_{1}=p$ is $B(n, p) D(n-p)$, where $D(m)$ is the number of permutations of the $m$ numbers $1,2, \cdots, m$ in which no number remains unmoved, i.e. there are no unit cycles. Then $D(m)$ is Euler's rencontre number and is the nearest integer to $m ! / e$ (see, for example, [3], [4], [9], [12]). Hence the number of $\pi$ for which $p_{1}=p$ is greater than $C n ! / p !$. Again, by (6), for such a $\pi$, we have $P_{1} \geqq \frac{1}{2} p(p-1)$ and so

$$
F_{\pi} \geqq B\left(P_{1}, q\right) \geqq B(p(p-1) / 2, q)=p ! \Lambda_{p}
$$

(say). Now $\log (p !)=O(p \log p)=O(q)$ and so

$$
\log \Lambda_{p}=2 q \log p-q \log q+O(q)=q \log q-2 q \log \log q+O(n) .
$$

Hence

$$
T_{1}(q) \geqq C(n !) \Lambda_{n} \geqq C(n !)\left\{q /(\log q)^{2}\right\}^{q} e^{-C^{n} n} .
$$

It follows from (8), (10) and (11) that

$$
T_{2}(q)=o\left(T_{1}(q)\right), \quad n ! T_{n q}=T_{1}(q)\{1+o(1)\} .
$$

This is true when $q+1$ replaces $q$ and so our lemma follows from (9).

We can improve the factor $n^{1 / 2}$ in (2) to $n /(\log n)^{2}$. More significantly, we can show that (1) holds over a range of $q$ of length $C_{1} n$ for any fixed positive $C_{1}$ and large enough $n$. It is possible that we can replace $C_{1} n$ by a constant multiple of $n \log n$. But these results require the development of a much more elaborate theory, and, in particular, a study of the asymptotic behaviour of $T_{n q}$ and of $T_{n, q \cdot 1} / T_{n q}$ for large $n$ and large $q$ such that $q<(n \log n) / 2$. 


\section{REFERENCES}

1. N. G. de Bruijn, Applied combinatorial mathematics, (ed. E. F. Beckenback), Wiley, New York, 1964, Chap. 5.

2. P. Erdös and A. Rényi, On random graphs. I, Publ. Math. Debrecen 6 (1959), 290-297. MR 22 \#10924.

3. L. Euler, Calcul de la probabilité dans le jeu de rencontre, Mem. Acad. Sci. Berlin 1753, 255-270; Opera Omnia (1) 7 (1923), 11-25.

4. - Solutio questionis curiosae ex doctrina combinationum, Mem. Acad. Sci. St. Petersbourg 3 (1811), 57-64; Opera Omnia (1) 7 (1923), 435-448.

5. F. Harary, The number of linear, directed, rooted, and connected graphs, Trans. Amer. Math. Soc. 78 (1955), 445-463. MR 16, 844.

6. - Graph theory, Addison-Wesley, Reading, Mass., 1969, pp. 214-223. MR 41 \#1566.

7. W. Oberschelp, Kombinatorische Anzahlbestimmungen in Relationen, Math. Ann. 174 (1967), 53-78. MR 36 \#1342.

8. G. Pólya, Kombinatorische Anzahlbestimmungen für Gruppen, Graphen und chemische Verbindungen, Acta Math. 68 (1937), 147-254.

9. J. Riordan, An introduction to combinatorial analysis, Wiley Publ. in Math. Statist., Wiley, New York; Chapman and Hall, London, 1958. MR 20 \#3077.

10. E. M. Wright, Asymptotic enumeration of connected graphs, Proc. Roy. Soc. Edinburgh Sect. A 68 (1968/69), 298-308. MR 42 \#1723.

11. - Graphs on unlabelled nodes with a given number of edges, Acta Math. 126 (1970), 1-9. MR 42 \#2975.

12. - Arithmetical properties of Euler's rencontre number, J. London Math. Soc. 4 (1972), 437--442.

Principal's Office, University of Aberdeen, Aberdeen, United Kingdom 